Abanico Agroforestal. Enero-Diciembre 2019;1:1-12. http://dx.doi.org/10.37114/abaagrof/2019.1 Artículo Revisión. Recibido: 18/02/2019. Aceptado: 19/11/2019. Publicado: 15/12/2019.

\title{
Selenio, selenoproteínas y estrés oxidativo en pequeños rumiantes. Revisión
} Selenium, selenoproteins and oxidative stress in small ruminants. Review

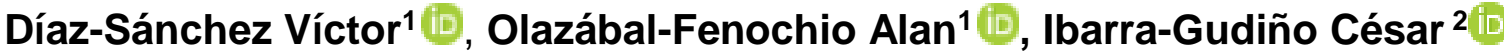

${ }^{1}$ Universidad Nacional Autónoma de México, Facultad de Estudios Superiores Cuautitlán, México. ${ }^{2}$ Facultad de Medicina Veterinaria y Zootecnia de la Universidad Autónoma de Nayarit; Nayarit, México. *Autor de correspondencia y responsable: Díaz-Sánchez Víctor. Universidad Nacional Autónoma de México. Facultad de Estudios Superiores Cuautitlán. Unidad de Investigación Multidisciplinaria. Cuautitlán Izcalli, Estado de México, México. C.P.54714.victorm_diazs@comunidad.unam.mx, cesaroctavio76@hotmail.com.

\section{RESUMEN}

La producción animal está condicionada por múltiples factores, dentro de estos, el más importante es la nutrición. Algunos de los problemas nutricionales más comunes en la producción están directamente relacionados con las características geográficas y las propiedades de los suelos en diferentes áreas de pastoreo y producción de forraje, un ejemplo de esto se observa en la pobre distribución natural de selenio en suelos de todo el mundo; una deficiencia que afecta a la producción, productos y subproductos de pequeños rumiantes. Los minerales, y específicamente, los microminerales como el selenio tienen un papel importante en la nutrición de los pequeños rumiantes, ya que, aunque no proporcionan energía, son esenciales para la síntesis de nutrientes, participando en numerosas actividades metabólicas como cofactores, manteniendo el efecto fisiológico y el equilibrio en el organismo. Las selenoproteínas son principalmente enzimas con capacidad antioxidante, que participan en el equilibrio oxidativo, eliminando los radicales libres que podrían dañar las células; causando enfermedades que afectan la función metabólica en el organismo animal, con un detrimento en la producción animal, por lo que la deficiencia debe ser corregida a través de la suplementación.

Palabras clave: selenio, selenoproteínas, estrés oxidativo, pequeños rumiantes.

\begin{abstract}
Animal production is conditioned by multiple factors, within these, the most important is nutrition. Some of the most common nutritional problems in production are directly related to the geographical characteristics and properties of soils in different areas of grazing and forage production, an example of this is observed in the poor natural distribution of selenium in soils throughout the world, a deficiency that affects the production, products and by-products of small ruminants. Minerals, and within these, microminerals such as selenium have an important role in the nutrition of small ruminants, since, although they do not provide energy, they are essential for the synthesis of nutrients, participating in numerous metabolic activities as cofactors, maintaining the physiological effect balance in the body. Selenoproteins are mainly enzymes with antioxidant capacity, which participate in the oxidative balance, eliminating free radicals that could damage cells, causing diseases that affect metabolic function in the animal organism, with a detriment in animal production, so that deficiency must be corrected through supplementation.
\end{abstract}

Keywords: selenium, selenoproteins, oxidative stress, small ruminants.

\section{INTRODUCCIÓN}

El selenio (Se) es un oligoelemento esencial para mantener el equilibrio fisiológico en los animales; actúa a través de proteínas con capacidad enzimática, que tienen 
principalmente propiedades antioxidantes en el organismo (Marek et al., 2013; Kruzhel et al., 2014). Las ovejas y cabras obtienen el mineral a través de la dieta (Tapiero et al., 2003; Slavica y Cepelak, 2004), por lo que es importante mencionar que el contenido de Se en el suelo por debajo de $0.010 \mathrm{mg} / \mathrm{kg}$ de materia seca puede causar signos de deficiencia en rumiantes; mientras que cantidades $<0.5 \mathrm{mg} / \mathrm{kg}$ en suelos $0<0.1 \mathrm{ng} / \mathrm{kg}$ en plantas se consideran insuficientes para la producción de selenoproteínas en el organismo (Ramírez-Bribiesca et al., 2001; Hefnawy y Tórtora-Pérez, 2010; Kruzhel et al., 2014); causando trastornos metabólicos y enfermedades relacionadas con la deficiencia del Se.

La deficiencia de Se, en asociación con la vitamina $\mathrm{E}$, produce la enfermedad del "músculo blanco". Los requerimientos de Se para los ovinos depende de la cantidad de vitamina E en la dieta; el nivel de Selenio sugerido para los ovinos es de $0.1 \mathrm{mg} \mathrm{Se} / \mathrm{kg}$ MS. Las experiencias obtenidas en México sugieren utilizar una dosis de $0.25 \mathrm{mg}$ Se en corderos aparentemente sanos y dosis de $0.5 \mathrm{mg}$ Se en corderos con signo de la distrofia muscular nutricional o enfermedad del músculo blanco (Carbajal et al., 2013).

Por lo anterior, es necesario realizar una suplementación en animales carentes del mineral para evitar estas patologías relacionadas con la deficiencia. El objetivo de este trabajo fue conjuntar la información más relevante con respecto al Se, la función de las selenoproteínas más importantes y su relación con el balance oxidativo.

\section{Absorción, distribución, metabolismo y excreción de selenio}

La absorción de Se en los rumiantes a través de la dieta puede variar del 11 al 35\% en promedio (Lescure et al., 2009; Hefnawy y Tórtora-Pérez, 2010; Kruzhel et al., 2014), debido a que el rumen, por sus características de $\mathrm{pH}$ y microbiota, pueden transformar el mineral principalmente a selenuros, que son formas químicas del elemento que el animal no puede absorber para la producción de selenoproteínas (Ghany-Hefnawy et al., 2007; Galbraith et al., 2015).

El mineral que pasa el rumen, llega al intestino delgado, donde se da la absorción de Se, principalmente en el duodeno y el íleon; los selenoaminoácidos, como la selenometionina, se absorben a través de la misma ruta utilizada por la metionina; a través del transporte activo, la selenocisteína se puede absorber de la misma manera, o se puede absorber unida a las proteínas; finalmente, las sales inorgánicas de Se son absorbidas por difusión pasiva en el tracto gastrointestinal (Juniper et al., 2009a; Rosen y Liu, 2009).

El metabolismo del selenato y/o el selenito en el organismo animal difieren entre sí. El selenato a nivel de rumen se puede reducir a selenito, debido al ambiente ruminal, 0 continuar a través del tracto gastrointestinal para ser absorbido en el intestino delgado por difusión pasiva. Por otro lado, el selenito también puede reducirse en el rumen, pero a diferencia del selenato, se reduce directamente a selenuros, que ni el animal ni la microbiota ruminal pueden utilizar para sus funciones metabólicas. 
Es importante señalar que tanto el selenato como el selenito, pueden ser utilizados por la microbiota ruminal para sintetizar selenometionina o selenocisteina, el cual puede ser utilizada por el animal a través de la digestión (Ghany-Hefnawy et al., 2007; Juniper et al., 2009a; Gresakova et al., 2013).

Después de la absorción, el Se puede usarse para la síntesis de selenoproteínas, almacenarse en forma de selenometionina, e insertarse de esta manera en diferentes proteínas en el organismo animal; o también ser excretado por diferentes vías (Juniper et al., 2009a). El Se es transportado a través del plasma, se incorpora a células como eritrocitos y leucocitos; así como a proteínas y enzimas como mioglobina, nucleoproteínas y miosina. A través del sistema se transporta al hígado, donde se mantiene una fracción del mineral dentro de los hepatocitos, el resto se trasladan por circulación a diferentes órganos, donde se almacenan como selenometionina, que puede incorporarse a las proteínas en lugar de a la metionina, pero sin actividad enzimática antioxidante (Gresakova et al., 2013). Dentro del organismo, la distribución de Se tiene una jerarquía, siendo la concentración más alta en riñones, seguida del hígado, el corazón y músculo esquelético (Berry, 2005; Qin et al., 2007; Juniper et al., 2009b; Birben et al., 2012).

Las selenoproteínas se forman dentro de células nucleadas a partir de compuestos orgánicos e inorgánicos. Independientemente de su origen, deben convertirse en selenuros, ya que es la forma química central para la formación de selenocisteina, único selenoaminoácido que puede insertarse en las selenoproteínas por su correcta función. A partir de este selenuro, puede haber dos rutas, una de las cuales es su metilación para su posterior excreción del organismo, o bien, su incorporación para la formación de selenocisteína, que se insertará en la formación de las diferentes selenoproteínas (Letavayová et al., 2006).

Tanto la fuente orgánica como la inorgánica de selenio se transforman en selenuro de hidrógeno $\left(\mathrm{H}_{2} \mathrm{Se}\right)$, compuesto central en la formación de selenoproteínas. En el caso de selenato, primero deberá reducirse a selenito, para luego reducirse a selenuro a través del glutatión. En el caso de la selenometionina, se transformará en selenuro de hidrógeno mediante una enzima-liasa, para luego incorporarla en proteínas como la selenometionina, o se transformará en selenocisteína mediante transulfuración, que se transformará en selenuro de hidrógeno mediante la enzima-liasa.

El selenuro $\left(\mathrm{H}_{2} \mathrm{Se}\right)$ será transformado por la enzima selenofosfato sintetasa en selenofosfato para la síntesis subsiguiente de selenocisteína, que se sintetiza en su tARN, que está inicialmente aminoacilado con serina, a través de la serina sintetasa, formando Ser-t-RNASec. Este residuo de serina proporciona el esqueleto de carbono de la selenocisteína; Ser-t-RNASec fosforila fosfoseril-t-RNASec, a través de la enzima PSTK (fosfoseril-t-RNASec quinasa); luego en una reacción que involucra la adición de selenofosfato en presencia de selenocisteína sintetasa; Phosphoseryl-t-RNASec se convierte en un Sec-t -RNASec, que se incorporará a las selenoproteínas a través de un codón UGA (Silva et al., 2000; Berry, 2005; Ohta y Suzuki, 2008). 
La excreción de Se puede ser por pulmones, heces y orina, dependiendo de la vía de administración y de los niveles tisulares. La administración de Se por vía oral tiene como vía principal de excreción a través de las heces; en caso de ingestión de altos niveles del mineral, las formas volátiles se espirarán a través de los pulmones. La inyección parenteral de Se será excretada principalmente a través de la orina. Finalmente, se sabe que la cantidad de mineral expulsado a través de la bilis es pequeña (Kuehnelt et al., 2007; Ohta y Suzuki, 2008; Kruzhel et al., 2014).

\section{Funciones de selenio}

Las funciones de este mineral se llevan a cabo a través de las selenoproteínas, que actúan de manera general en las reacciones de oxidación-reducción, con el objetivo de mantener el equilibrio oxidativo (Köhrle, 2004; Lescure et al., 2009; Rahmanto y Davies, 2012; Kruzhel et al., 2014). De esta manera, el Se participa en la respuesta inmune, espermatogénesis, procesos de crecimiento y desarrollo, la defensa contra el daño oxidativo y la regulación de las hormonas tiroideas; así como también indirectamente en la regulación y eficiencia de los procesos productivos (Ramírez-Bribiesca et al., 2004; Revilla-Vázquez, et al., 2008; Kumar et al., 2009). Contrariamente, concentraciones bajas de Se tienen relación con problemas productivos y reproductivos en pequeños rumiantes (Celi, 2010; Mahmoud et al., 2013). La deficiencia en pequeños rumiantes puede deberse a una restricción en la dieta o una baja absorción en el tracto gastrointestinal. La deficiencia de Se es la causa principal de varias enfermedades, como: distrofia muscular nutricional, anemia, mastitis, infertilidad asociada con baja calidad seminal, retención de placenta o productos y abortos, baja inmunidad, animales débiles, y menores ganancias de peso, producción de leche y lana (Forman y Torres 2002; Sobiech y Kuleta, 2002; Arthur et al., 2003; Gill y Walker, 2008; Lekatz et al., 2010; Haenlein y Anke, 2011; Karami et al., 2011; Pavlata et al., 2012; Stefanowicz et al., 2013).

\section{Distrofia muscular nutricional o enfermedad de músculo blanco}

Es el principal signo causado por la deficiencia de Se, consecuencia de un daño en las membranas de los miocitos; se puede presentar en la mayoría de los animales, aunque de modo más frecuente en animales jóvenes, cuyas madres consumieron una ración carente de Se durante la gestación (Ramírez-Bribiesca et al., 2001; Sobiech y Kuleta, 2002). En animales selenodeficientes, los fosfolípidos de la membrana de los miocitos sufren una peroxidación, causando daño y mal funcionamiento a las proteínas de la membrana. La principal consecuencia de la lesión celular es el incremento de la permeabilidad de calcio, el cual se acumula causando daño, y por lo tanto, pérdida de la funcionalidad muscular (Karami et al., 2011).

La distrofia muscular se puede manifestar de manera aguda o crónica; la forma aguda se caracteriza por muerte del animal en pocas horas. Los signos comúnmente observados son anormalidades al caminar, temblor y disminución del tono muscular y alteraciones 
cardiacas. En zonas con selenodeficiencia se considera la principal causa de muerte en pequeños rumiantes (Ramírez-Bribiesca et al., 2001).

\section{Anemia}

En animales selenodeficientes, se ha observado anemia y cuerpos de Heinz asociados a la baja actividad de las enzimas antioxidantes de los eritrocitos y la consecuente destrucción celular (Stefanowicz et al., 2013).

\section{Mastitis}

La deficiencia de Se y la baja actividad de la selenoenzima glutatión peroxidasa, condicionan la actividad fagocítica de los leucocitos en enfermedades como la mastitis, causando proliferación bacteriana y daño a la glándula mamaria. Se ha demostrado que la suplementación con este micronutriente aumenta la eficacia de la fagocitosis por parte de neutrófilos y macrófagos, eliminando de forma eficaz a los patógenos que invaden la glándula mamaria (Sánchez et al., 2006).

\section{Retención placentaria}

La selenodeficiencia puede llevar a un incremento en la retención placentaria. De forma normal el tejido placentario ejerce una fuerte actividad quimiotáctica sobre los leucocitos, los cuales tienen que ver con la separación de la placenta después del parto. Si la actividad quimiotáctica sobre los leucocitos es deficiente o retardada, se producirá la retención placentaria, la cual contribuye a la infección del útero, dañando la capacidad de gestación (Lekatz et al., 2010).

\section{Respuesta inmune comprometida}

La deficiencia de Se es bien conocida por deprimir tanto la respuesta inmune humoral, como la respuesta celular. Se ha observado el aumento de la lgG calostral en rumiantes suplementados, así como un aumento en la respuesta de los anticuerpos en crías suplementadas con Se (Rose et al., 2012). Kumar et al., encontraron que al suplementar ovejas con Se y después exponerlas a un agente bacteriano, la respuesta inmune humoral aumentó significativamente con respecto al grupo testigo (Kumar et al., 2009). Los fagocitos contienen glutatión peroxidasa (GSHPx) en los lisosomas. Un déficit nutricional de Se produce la declinación de la actividad de la GSHPx en estas células, y por lo tanto en la habilidad para destruir al antígeno fagocitado (Forman y Torres, 2002; Hoffmann y Berry, 2008; Avery y Hoffmann, 2018).

\section{Funciones de selenio y balance oxidativo}

El Selenio se relaciona principalmente con el equilibrio oxidativo, ya que la mayoría de las selenoproteínas tienen una función antioxidante en el organismo (Hawkes y Alkan, 2010). Cuando hay un aumento en los radicales libres que no pueden ser regulados por los sistemas enzimáticos y las moléculas antioxidantes, atacan las membranas celulares, 
alterando su función y viabilidad. El desequilibrio entre oxidantes y antioxidantes a favor de los primeros se denomina "estrés oxidativo", debido a la alta producción de especies reactivas de oxígeno $(E R O)$, la cuales en altas concentraciones pueden dañar el funcionamiento celular. Factores como la contaminación, radiación, medicamentos, infecciones y la inflamación, generan estrés oxidativo; causando un exceso de ERO en el organismo (Forman y Torres, 2002), que dañan específicamente las estructuras de los carbohidratos, ácidos nucleicos, lípidos y proteínas; alterando su función y causando daños a la estructura celular, que tendrá un impacto en la función correcta de los órganos y la homeostasis del organismo (Grotto et al., 2009; Rahmanto y Davies 2012; Birben et al., 2012).

Tabla 1. Principales selenoproteínas y su función

Selenoproteina

Familia Glutatión peroxidasa (GPx1, GPx2, GPx3, GPx4, GPx6; snGPx-4)

Familia Thioredoxin reductasa (TRx1,2,3; TGR)

Familia de Deiodinasas

(SPS2)

Selenoproteinas de 15 y 18 $\mathrm{kDa}$ (Sell5, Sell8)

Selenoproteína M (SelM)

Selenoproteína N (SeIN)

Selenoproteína P (SelP)

Selenoproteína R (SeIR, MsrB)

Selenoproteína T (SelT)

Selenoproteína W (SelW)

Selenoproteína H, K, M, O, S,

$\mathrm{V}, \mathrm{Y}, \mathrm{Z}$

Selenoproteína I

Distribución en tejido

Obicua (GPx1, GPx4), gastrointestinal (GPx2),

Riñón y plasma (GPx3), Epitelio olfatorio, Glándula de Bowman (GPx6), testis, Esperma (snGPx4)

Todas las células del organismo

Expresión y regulación en tejidos específicos, principalmente en tiroides, hígado, riñón y pituitaria

Varios tejidos

\section{Varios tejidos, cerebro}

Varios tejidos, cerebro

Músculos esquelético, hígado, riñón, corazón, estómago

El hígado es la principal fuente de SelP; obicua Varios tejidos

Músculo esquelético, músculo cardiaco, cerebro, testículo e hígado

Músculo esquelético, músculo cardiaco, cerebro, testículo e hígado

Varios tejidos

Varios tejidos
Antioxidante, modulación de lipoxygenasas, transducción de señales redox

Regulación redox, metabolismo de drogas, transducción de señales

Catalizan la conversión de T4 a T3 y la degradación de rT3 (DIO1 y DIO2) así como T4 y T3 (DIO1 y DIO3)

Cataliza la producción de selenofosfato, se requiere para la incorporación de selenocisteína dentro de las selenoproteínas

Desconocida

Proliferación y regeneración

Mutaciones que llevan a espina rígida y distrofia muscular

Transporte de selenio, antioxidante

Reductasa sulfoxido R-metionina

Antioxidante

Antioxidante

Desconocida

Hipotético fosfatidil transferasa CDPalcohol

(Modificado de Holben y Smith, 1999; Köhrle, 2004; Kumar y Priyadarsini, 2014)

Para contrarrestar las ROS, existen diferentes líneas de defensa en el cuerpo, que se controlan mediante un sistema que incluye eliminadores de radicales oxidantes de bajo peso molecular, enzimas y defensas no enzimáticas (Rahmanto y Davies, 2012). Actualmente, se conocen aproximadamente 30 selenoproteínas en los mamíferos, dentro de las cuales la mayoría tiene funciones antioxidantes. Ver tabla 1. (Haenlein y Anke, 2011). 


\section{Métodos de suplementación de selenio}

Debido a las condiciones del suelo en todo el mundo y las características fisiológicas de los rumiantes, es posible que exista una deficiencia de Se en ovejas y cabras, por lo que será necesario realizar una suplementación para evitar problemas. La suplementación en pequeños rumiantes tiene múltiples beneficios en la producción animal. Esta se realiza a través de sales minerales orgánicas o inorgánicas, que pueden incorporarse en la dieta, agua, bloques de mezcla de minerales, soluciones inyectables o formas de liberación prolongada como bolos intrarruminales (Hall et al., 2009; López-Arellano et al., 2015). La suplementación del mineral se reflejará en el organismo, donde los niveles de Se en sangre pueden ser un indicador de la correcta suplementación de este elemento en los rumiantes (intravitalmente), así como la medición de la actividad de las selenoproteínas, o de los niveles en los órganos. Los niveles deficientes, adecuados y tóxicos de Se están establecidos en tejidos de pequeños rumiantes, ver tabla 2.

Tabla 2. Niveles de selenio en sangre, hígado, riñón, músculo y leche en pequeños rumiantes

\begin{tabular}{c|c|c|c|c|c}
\hline & Sangre & Hígado & Riñón & Músculo & Leche \\
\hline Deficientes & $0.003-0.040$ & $0.01-0.10$ & $0.05-0.60$ & $0.01-0.03$ & $0.002-0.020$ \\
Marginales & $0.040-0.080$ & $0.15-0.25$ & $0.70-1.10$ & $0.03-0.09$ & $0.020-0.030$ \\
Adecuados & $0.150-0.500$ & $0.25-1.50$ & $0.90-3.00$ & $0.09-0.40$ & $0.025-0.250$ \\
$\quad$ Altos & - & $2.00-10.0$ & $4.00-6.00$ & $0.40-0.60$ & - \\
Tóxicos & - & $15.0-30.00$ & $6.00-15.00$ & $0.60-20.00$ & - \\
\hline
\end{tabular}

ppm peso húmedo. Puls, 1988.

Se considera una dosis terapéutica preventiva de $0.25 \mathrm{mg} / \mathrm{kg}$ de peso vivo por vía parenteral (Ramírez-Bribiesca et al., 2005). Se recomienda suplementar a los machos y hembras 3-4 semanas antes de la temporada de apareamiento y 3-4 semanas antes del parto. De la misma manera, se recomienda suplementar a los corderos y cabritos al nacer, al destete y al comienzo del engorde. En el caso de la suplementación con dieta, se recomienda hacer una dosis de 1-3 ppm de acuerdo con la administración federal de medicamentos de la Food and Drug Administration (FDA, 2007).

\section{Signos de toxicidad por selenio ingerido}

Si se excede la dosis de Se requerida por el animal, puede haber riesgo de intoxicación. Los principales signos de intoxicación moderada son: anorexia, rechinamiento de los dientes, secreción de moco por la nariz y membranas mucosas cianóticas. En la intoxicación aguda, el animal presentará hemorragias diseminadas en el cuerpo y muerte súbita (Revilla-Vázquez et al., 2008).

\section{CONCLUSIÓN}

El selenio es fundamental para todos los animales, permite mantener el balance oxidativo a través de las selenoproteínas, distribuidas en el organismo. Existen en el mundo suelos con deficiencia de este mineral, por lo que es importante la suplementación, principalmente en rumiantes; ya que estos absorben en proporción menores cantidades 
del mineral, a diferencia de los animales no rumiantes. La suplementación se podrá hacer a través de diferentes vías, tomando en cuenta las dosis recomendadas para evitar la intoxicación de los animales.

\section{LITERATURA CITADA}

AVERY JC y Hoffmann PR. 2018. Selenium, Selenoproteins, and Immunity. Nutrients. 1203 (10): 1-20. https://doi.org/10.3390/nu10091203

ARTHUR JR, Mckenzie RC, Beckett GJ. 2003. Immunity Enhanced by Trace Elements. American Society for Nutritional Sciences. 133: 457-459. https://doi.org/10.1677/joe.1.05971

BERRY MJ. 2005. Insights into the hierarchy of selenium incorporation. Nat. Genet. 37(11): 1162-1163. https://doi.org/10.1038/ng1105-1162

BIRBEN E, Sahiner UM, Sackesen C, Erzurum S, Kalayci O. 2012. Oxidative stress and antioxidant defense. World Allergy Organ. J. 5(1): 9-19. https://doi.org/10.1097/WOX.0b013e3182439613

CARBAJAL Hermosillo MA, Aquí Quintero G, Díaz Gutiérrez C. 2013. Uso de selenio en ovinos. Abanico Veterinario. 3(1):44-54.

https://biblat.unam.mx/hevila/Abanicoveterinario/2013/vol3/no1/5.pdf

CELI P. 2010. The role of oxidative stress in small ruminants' health and production. Rev. Bras. Zootec. 39: 348-363. http://dx.doi.org/10.1590/S1516-35982010001300038

FOOD and Drug Administration. U.S. 2007. Department of Health and Human Services. http://www.fda.gov/. Fecha de consulta, 2 de Febrero 2019

FORMAN HJ y Torres M. 2002. Reactive oxygen species and cell signaling: Respiratory burst in macrophage signaling. Am. J. Respir. Crit. Care Med. 166: S4-S8. https://doi.org/10.1164/rccm.2206007

GALBRAITH ML, Vorachek WR, Estill CT, Whanger PD, Bobe G, Davis TZ, HALL J. 2015. Rumen Microorganisms Decrease Bioavailability of Inorganic Selenium Supplements. Biol. Trace Elem. Res. 7-12. https://doi.org/10.1007/s12011-015-0560-8

GHANY-HEFNAWY AEL, López-Arellano R, Revilla-Vázquez A, Ramírez-Bribiesca E, Tórtora-Pérez J. 2007. The relationship between fetal and maternal selenium concentrations in sheep and goats. Small Rumin. Res. 73: 174180. https://doi.org/10.1016/j.smallrumres.2007.01.020 
GILL HS y Walker GP. 2008. Selenium, immune function and resistance to viral infections. Nutr. Diet. 65:S41-S47. https://doi.org/10.1111/j.1747-0080.2008.00260.x

GRESAKOVA L, Cobanova K, Faix S. 2013. Selenium retention in lambs fed diets supplemented with selenium from inorganic or organic sources. Small Rumin. Res. 111(13): 76-82. https://doi.org/10.1016/j.smallrumres.2012.10.009

GROTTO D, Santa Maria L, Valentini J, Paniz C, Schmitt G, Garcia SC, Juarez Pomblum V, Rocha JB, Farina M. 2009. Importance of the lipid peroxidation biomarkers and methodological aspects for malondialdehyde quantification. Quim. Nova. 32(1): 169-174. http://dx.doi.org/10.1590/S0100-40422009000100032

HAENLEIN GFW y Aanke M. 2011. Mineral and trace element research in goats: A review. Small Rumin. Res. 95(1): 2-19. https://doi.org/10.1016/j.smallrumres.2010.11.007

HALL JA, Van Saun RJ, Nichols T, Mosher W, Pirelli G. 2009. Comparison of selenium status in sheep after short-term exposure to high-selenium-fertilized forage or mineral supplement. Small Rumin. Res. 82(1): 40-45. https://doi.org/10.1016/j.smallrumres.2009.01.010

HAWKES WC y Alkan Z. 2010. Regulation of redox signaling by selenoproteins. Biol. Trace Elem. Res. 134(3): 235-251. https://doi.org/10.1007/s12011-010-8656-7

HEFNAWY AEG y Tórtora-Pérez JL. 2010. The importance of selenium and the effects of its deficiency in animal health. Small Rumin. Res. 89(2-3): 185-192. https://doi.org/10.1016/j.smallrumres.2009.12.042

HOFFMANN PR y Berry MJ. 2008. The influence of selenium on immune responses. $\mathrm{Mol}$ Nutr Food Res. 52(11): 1273-1280. https://doi.org/10.1002/mnfr.200700330

HOLBEN DH y Smith AM. 1999. The diverse role of selenium within selenoproteins: A review. J. Am. Diet. Ass. 99: 836-843. https://doi.org/10.1016/S0002-8223(99)00198-4

JUNIPER DT, Phipps RH, Ramos-Morales E, Bertin G. 2009a. Effect of high dose selenium enriched yeast diets on the distribution of total selenium and selenium species within lamb tissues. Livest. Sci. 122(1): 63-67. https://doi.org/10.1016/j.livsci.2008.07.025

JUNIPER DT, Phipps RH, Ramos-Morales E, Bertin G. 2009b. Effects of dietary supplementation with selenium enriched yeast or sodium selenite on selenium tissue distribution and meat quality in lambs. Anim. Feed Sci. Technol. 149(3-4): 228-239. http://dx.doi.org/10.1016/j.anifeedsci.2008.06.009 
KARAMI M, Alimon AR, Sazili AQ, Goh YM, Ivan M. 2011. Effects of dietary antioxidants on the quality, fatty acid profile, and lipid oxidation of longissimus muscle in Kacang goat with aging time. Meat Sci. 88(1): 102-108. https://doi.org/10.1016/j.meatsci.2010.12.009

KÖHRLE, J. 2004. Selenium in biology and medicine-further progress and increasing interest. Journal of Trace Elements in Medicine and Biology. 18(1): 61-63. http://doi.org/10.1016/j.jtemb.2004.06.001

KRUZHEL B, Vovk S, Malgorzata B, Nowakowska E, Sergei P. 2014. Selenium in the diet of ruminants. Acta Sci. Pol., Zootech. 13(4): 5-16. http://asp.zut.edu.pl/2014/13_4/asp2014-13-4- 256.pdf

KUEHNELT D, Juresa D, Francesconi KA, Fakih M, Reid ME. 2007. Selenium metabolites in urine of cancer patients receiving L-selenomethionine at high doses. Toxicol. Appl. Pharmacol. 220(2): 211-5. https://doi.org/10.1016/j.taap.2007.01.005

KUMAR N, Garg AK, Dass RS, Chaturvedi VK, Mudgal V, Varshney VP. 2009. Selenium supplementation influences growth performance, antioxidant status and immune response in lambs. Anim. Feed Sci. Technol. 153(1-2): 77-87. https://doi.org/10.1016/j.anifeedsci.2009.06.007

KUMAR BS y Priyadarsini KI. 2014. Selenium nutrition: How important is it?. Biomedicine \& Preventive Nutrition. 4(2): 333-341. https://doi.org/10.1016/j.bionut.2014.01.006

LEKATZ LA, Ward MA, Borowicz PP, Taylor JB, Redmer DA, Grazul-Bilskal AT, Reynolds LP, Caton JS, Vonnahme KA. 2010. Cotyledonary responses to maternal selenium and dietary restriction may influence alterations in fetal weight and fetal liver glycogen in sheep. Anim. Reprod. Sci. 117(3-4): 216-25. https://doi.org/10.1016/j.anireprosci.2009.05.009

LESCURE A, Rederstorff M, Krol A, Guicheney P, ALLAMAND V. 2009. Selenoprotein function and muscle disease. Biochim. Biophys. Acta. 1790(11): 1569-74. https://doi.org/10.1016/j.bbagen.2009.03.002

LETAVAYOVÁ L, VIcková V, Brozmanová J. 2006. Selenium: from cancer prevention to DNA damage. Toxicology. 227(1-2):1-14. https://doi.org/10.1016/j.tox.2006.07.017

LÓPEZ-ARELLANO R, Ramírez-Bribiesca JE, Jaimes-Miranda J, Tortora-Pérez JL, Revilla-Vazquez AL, Rodríguez-Patiño G, Montaño-Gomez MF. 2015. Pathophysiological 
response to experimental oral overdose of different forms of selenium in lambs. Ann. Anim. Sci. 15(3):655-666. https://doi.org/10.1515/aoas-2015-0016

MAHMOUD GB, Abdel-Raheem SM, Hussein HA. 2013. Effect of combination of vitamin $E$ and selenium injections on reproductive performance and blood parameters of Ossimi rams. Small Rumin. Res. 113(1): 103-108. https://doi.org/10.17582/journal.aavs/2019/7.6.434.440

MAREK K y Stanislaw B. 2013. Selenium: Significance, and outlook for supplementation. Nutrition. 29: 713-718. https://doi.org/10.1016/j.nut.2012.11.012.

OHTA Y y Suzuki KT. 2008. Methylation and demethylation of intermediates selenide and methylselenol in the metabolism of selenium. Toxicol. Appl. Pharmacol. 226(2): 169-77. https://doi.org/10.1016/j.taap.2007.09.011

PAVLATA L, Mišurová L, Pechová A, Dvořák R. 2012. Comparison of organic and inorganic forms of selenium in the mother and kid relationship in goats. Czech J. Anim. Sci. 57(8): 361-369. https://doi.org/10.17221/6271-CJAS

PULS R. 1988. Mineral levels in animal health diagnostic data. Sherpa International, USA. ISBN 0-9693429-0-X

QIN S, Gao J, Huang K. 2007. Effects of different selenium sources on tissue selenium concentrations, blood GSH-Px activities and plasma interleukin levels in finishing lambs. Biol. Trace Elem. Res. 116(1): 91-102. https://doi.org/10.1007/BF02685922

RAHMANTO AS y Davies MJ. 2012. Selenium-containing amino acids as direct and indirect antioxidants. IUBMB Life. 64(11):863-871. https://doi.org/10.1002/iub.1084

RAMÍREZ-BRIBIESCA E, Hernández-Camacho E, Hernández-Calva LM, Tórtora-Pérez JL. 2004. Efecto de un suplemento parenteral con selenito de sodio en la mortalidad de corderos y los valores hemáticos de selenio. Agrociencia. 38(1): 43-51. ISSN: 14053195. https://www.redalyc.org/articulo.oa?id=30238105

RAMÍREZ-BRIBIESCA JE, Tórtora JL, Hernández LM, Huerta M. 2001. Main causes of mortalities in dairy goat kids from the Mexican plateau. Small Rumin. Res. 41: 77-80. https://doi.org/10.1016/S0921-4488(01)00191-2

RAMÍREZ-BRIBIESCA JE, Tórtora JL, Huerta M, Aguirre A, Hernández LM. 2001. Diagnosis of selenium status in grazing dairy goats on the Mexican plateau. Small Rumin. Res. 41(1): 81-85. https://doi.org/10.1016/S0921-4488(01)00188-2 
RAMÍREZ-BRIBIESCA JE, Tórtora-Pérez J, Hernández LM, López R, Crosby M. 2005. Effect of selenium-vitamin $E$ injection in selenium-deficient dairy goats and kids on the Mexican plateau. Arq. Bras. Med. Vet. Zootec. 57(1): 77-84. https://doi.org/10.1590/S0102-09352005000100011

REVILLA-VÁZQUEZ A, Ramírez-Bribiesca E, López-Arellano R, Hernández-Calva LM, Tórtora-Pérez JL, García-García E, Cruz-Monterrosa RG. 2008. Suplemento de selenio con bolos intrarruminales de selenito de sodio en ovinos. Agrociencia. 42: 629-635. ISSN: $\quad 14053195$. http://www.scielo.org.mx/scielo.php?pid=S140531952008000600003\&script=sci_abstract

ROSE M, Pearson S, Cratchley T. 2012. Effect of iodine, selenium and cobalt rumen boluses given to dry dairy cows on the immunoglobulin and thyroid hormone status of calves. Anim. Sci. J. 83(7): 543-8. https://doi.org/10.1111/j.1740-0929.2011.00991.x

ROSEN BP y Liu Z. 2009. Transport pathways for arsenic and selenium: A minireview. Environ. Int. 35(3): 512-515. https://doi.org/10.1016/j.envint.2008.07.023

SÁNCHEZ J, Montes P, Jiménez A, Andrés S. Prevention of Clinical Mastitis with Barium Selenate in Dairy Goats from a Selenium-Deficient Area. J. Dairy Sci. 90:2350-2354. https://doi.org/10.3168/jds.2006-616

SILVA JH, Quiroga MA, Auza NJ. 2000. Selenio en el rumiante. Relaciones suelo, planta, animal. Med. Vet. 17(10): 229-246. ISSN 0212-8292. https://dialnet.unirioja.es/ejemplar/342778

SLAVICA D y Cepelak I. 2004. The facts and controverses about selenium. Acta Pharm. (54): 261-276. PMID: 15634611 https://www.researchgate.net/publication/8095899

STEFANOWICZ FA, Talwar D, O'reilly DSJ, Dickinson N, Atkinson J, Hursthouse AS, Rankin JDA. 2013. Erythrocyte selenium concentration as a marker of selenium status. Clin. Nutr. 32(5): 837-842. https://doi.org/10.1016/j.clnu.2013.01.005

SOBIECH P y Kuleta Z. 2002. Usefulness of some biochemical indicators in detection of early stages of nutritional muscular dystrophy in lambs. Small Rumin. Res. 45(2): 209215. https://doi.org/10.1016/S0921-4488(02)00102-5

TAPIERO H, Townsend D, Tew K. 2003. The antioxidant role of selenium and selenocompounds. Biomed. Pharmacother. 57(1-3): 134-144. PMCID: PMC6361120 